\title{
SoftiMAX - A new Soft X-ray Microscopy and Coherent Imaging Beamline at the MAX IV Facility.
}

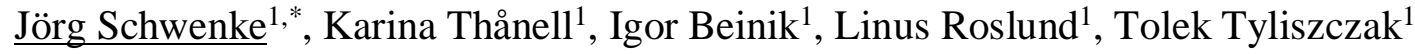 \\ 1. MAX IV Laboratory, Lund University, Lund, Sweden. \\ * Corresponding author, jorg.schwenke@maxiv.lu.se
}

SoftiMAX is a new beamline for soft X-ray spectro-microscopy currently under construction at the new MAX IV synchrotron facility in Lund, Sweden. It is situated at the $3 \mathrm{GeV}$ ring at MAX IV, and will provide a very high average coherent flux owing to the low emittance properties of the ring [1]. The SoftiMAX project consists of two branch lines, utilizing different focusing solutions and catering to a variety of imaging methods. As the construction of the beamline is nearing completion, we present the design and an outlook on the capabilities of the experimental stations when the beamline comes into operation in 2019.

In overview, the available photon energy range of SoftiMAX is 275 to $2500 \mathrm{eV}$, with full polarization control up to approx. $1650 \mathrm{eV}$. The energy resolution will lie between 1000 and $15000 \mathrm{E} / \mathrm{dE}$, depending on the grating and energy used, and the flux on sample in the main branch end-station is ca. $2 \times 10^{10} \mathrm{ph} / \mathrm{sec}$ at $275 \mathrm{eV}$ and ca. $2 \times 10^{9} \mathrm{ph} / \mathrm{sec}$ at $2500 \mathrm{eV}$ with a $1200 \mathrm{l} / \mathrm{mm}$ grating and a $20 \mathrm{~nm}-\mathrm{FZP}$.

The main branch of Soft $i$ MAX will host an end-station optimized for scanning techniques such as Scanning Transmission X-ray Microscopy (STXM) and diffraction imaging (ptychography [2]). Accordingly, the x-ray beam will be focused using Fresnel Zone Plates (FZP) to a beam size on the sample below $100 \mathrm{~nm}$ to allow for small illumination footprint (STXM) or a larger beam size to ease methods that require overlapping illumination (ptychography). STXM will be the first method offered when SoftiMAX becomes operational in early 2019, and the STXM branch is envisioned to support a wide range of sample types, including material science, environmental science, life science and biology.

In our microscope design, the sample is fixed on the beam axis, and the FZP element (and OSA) is moved for focusing. Both the FZP and the sample scanner assembly can be used for unidirectional continuous line scanning during data acquisition. The scanner control loop is closed by an interferometer setup and will have a maximum field-of-view of approximately $80 \mu \mathrm{m}$, while the dwell times are planned to be as short as $100 \mu$ s per pixel. The first version of the sample holder will operate at room temperature, and can be used in concert with a PMT detector, a silicon drift (fluorescence) detector, a avalanche photodiode, and a sCMOS pixel detector for high-resolution diffraction imaging with an acquisition rate of up to 100 fps. The sample assembly is exchangeable and can be replaced with a custom sample environment.

The second branch of SoftiMAX will provide a larger spot size than the main branch, but higher flux, by using Kirkpatrick-Baez focusing optics. The maximum total flux is estimated to be $5 \times 10^{13} \mathrm{ph} / \mathrm{sec}$ at $900 \mathrm{eV}$ in a spot of $20 \mu \mathrm{m}$ in the focal plane (300 1/mm grating). The micrometer beam size will support methods that require a fully coherent extended beam, such as Fourier Transform Holography, which is the principal mode of operation for this branch. The corresponding end-station will be optimized for the studies of magnetic thin films using magnetic dichroism, but is still in the planning stage.

Moreover, support for Resonant Soft X-ray scattering (RSoXS [3]) and X-ray Resonant Magnetic 
Scattering (XRMS [4]) is planned, which will further utilize the variable polarization of the x-rays, and allow us to explore the effects of coherence on these methods. The energy range at SoftiMAX is complimentary to hard X-ray beamlines in this case, as it allows the study of meso-scale structures. The long working distance between the focusing optics and the focal plane allows for more complicated sample environments than on the main branch. For example, a static magnetic field setup with a field strength of up to $0.8 \mathrm{~T}$ is being developed in collaboration with the SEXTANTS beamline at the SOLEIL synchrotron [5]. The second branch end-station(s) can be built to be interchangeable. For example, we envision to implement an end-station with rotatory motion for the sample and detector for reflectometry experiments or imaging schemes. User end-stations can also be supported. The second branch will be operational in 2020 at the earliest.

The SoftiMAX beamline is part of the Imaging group at MAX IV, and embedded in a wider infrastructure for sample preparation and data analysis, including a dedicated computer cluster and in-house support for spectral analysis and phase retrieval of diffraction data.

The installation of the insertion device and beamline optics will take place over summer and fall of 2018, and first user operation on the main branch is expected to start in early 2019 [6].

\section{References:}

[1] https://www.maxiv.lu.se/accelerators-beamlines/accelerators/accelerator-documentation/3-gevstorage-ring/

[2] P. Thibault et al, PNAS 113 (2016) E8219.

[3] http://gisaxs.com/index.php/RSoXS

[4] D. Raoux et al, ACTA PHYS POL A 98 (2000) 5.

[5] https://www.synchrotron-soleil.fr/fr/lignes-de-lumiere/sextants

[6] The SoftiMAX project is funded by the Swedish Research Council.

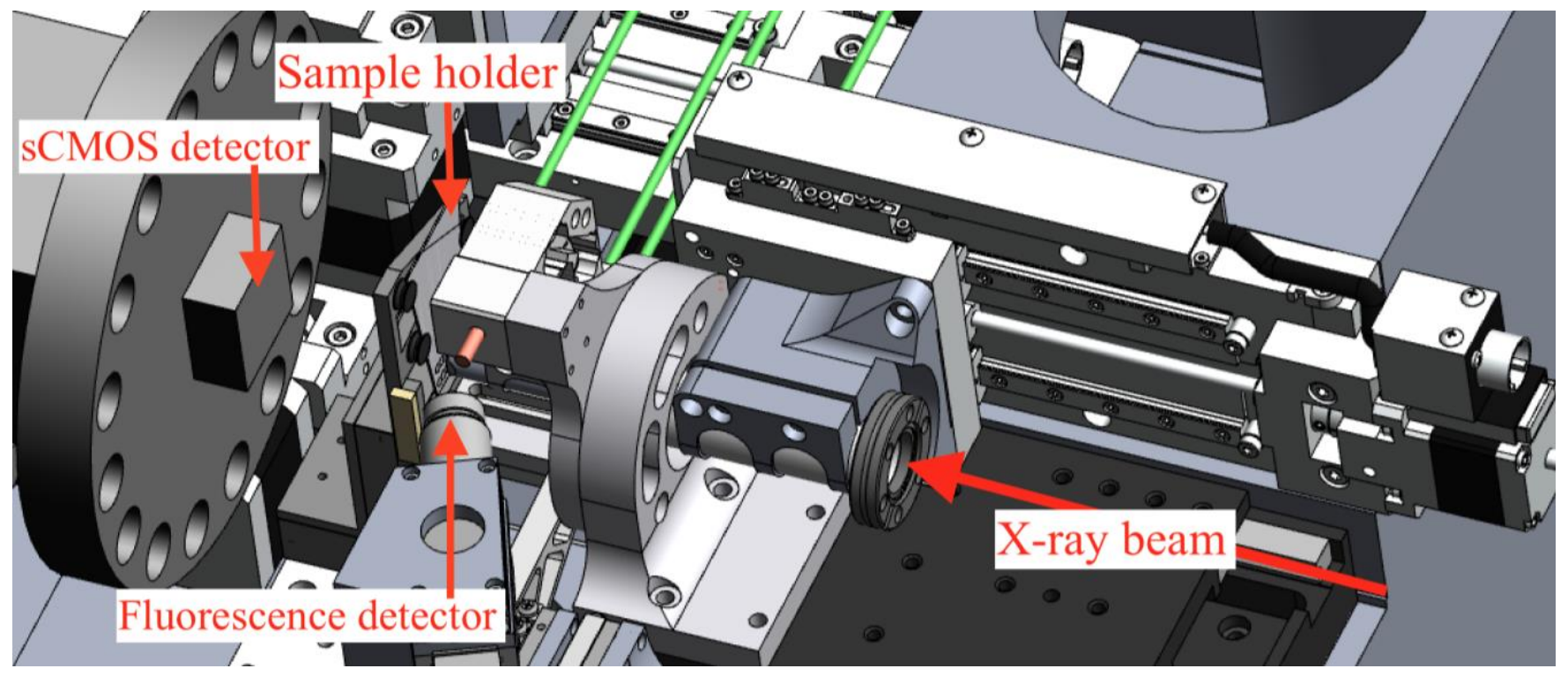

Figure. 1. SoftiMAX-STXM end-station on the main branch. The drawing shows the design of the sample scanner assembly and the focusing column. The sCMOS pixel detector and the fluorescence detector are shown, and the interferometer beams are drawn in green colour. 